\title{
Importance of Laryngopharyngeal Reflux in the Incidence of Benign Vocal Folds' Lesions
}

\author{
Anna Salwa
}

\begin{abstract}
Introduction: The laryngopharyngeal reflux (LPR) is defined as the retrograde flow of gastric contents to the upper respiratory tract causing mucosal damage.

Objectives: The study aims to determine the relationship between the incidence of LPR and the presence of benign lesions of the vocal folds. Materials and methods: Using the reflux finding score (RFS), we determined the incidence of the LPR in patients with changes such as Reinke's edema, polyps, and granulomas of the vocal folds.

Results: The prevalence of pathologic LPR was $25 \%$ in the control group, $81 \%$ in the Reinke's edema group, $60 \%$ in the vocal folds granulomas group, and $41 \%$ in the vocal cord polyps group. The most common manifestation of the LPR in the videolaryngoscopic examination is hypertrophy of the posterior commissure, which is anatomically explained by the closest location to the esophagus.

Conclusion: In conclusion, the LPR might be an etiologic factor in Reinke's edema, polyps, and granulomas of the vocal folds.

Keywords: Granuloma, Laryngopharyngeal reflux, Reflux finding score, Reinke's edema, Vocal cord polyps.

International Journal of Phonosurgery \& Laryngology (2019): 10.5005/jp-journals-10023-1168
\end{abstract}

\section{INTRODUCTION}

Approximately, $15 \%$ of patients presenting in an otolaryngologist's office is suffering from laryngopharyngeal reflux (LPR). ${ }^{1,2}$ Patients usually present completely different symptoms than those in the gastroesophageal reflux (GERD). According to Koufman, most common symptoms are dysphonia or hoarseness, cough, foreign body sensation in the throat, throat clearing, and dysphagia. ${ }^{1,3,4}$ Acid reflux of gastric contents into the larynx contributes to the relatively rapid deterioration of the mucosa due to less developed defense mechanisms as compared with the esophageal mucosa. ${ }^{1,3}$ The LPR can manifest itself in different ways during videolaryngoscopic examination. To assess the severity of the LPR, Belafsky developed the reflux finding score (RFS). The parameters included in the scale are presented in Table 1. The patients with scores above seven points have a $95 \%$ probability of presenting LPR. ${ }^{1,5}$ The scale has high reproducibility and can be used to assess the effectiveness of antireflux treatment. ${ }^{5}$ The study aimed to determine the relationship between LPR and the occurrence of changes such as Reinke's edema, vocal cord polyps, and laryngeal granulomas.

Table 1: Reflux finding score-point value from 0 to 26 , RFS $>7=$ LPRD

\begin{tabular}{ll}
\hline Laryngoscopic findings & Score \\
\hline Subglottic edema & 0-absent, 2-present \\
Ventricular obliteration & 2-partial, 4-complete \\
Erythema/hyperemia & 2-arytenoids only, 4-diffuse \\
Vocal fold edema & 1-mild, 2-moderate, \\
& 3-severe, 4-polypoid \\
Diffuse laryngeal edema & 1-mild, 2-moderate, \\
& 3-severe, 4-obstructing \\
Posterior comissure hypertrophy & 1-mild, 2-moderate, \\
& 3-severe, 4-obstructing \\
Granuloma/granulation & 0-absent, 2-present \\
Thick endolaryngeal mucus & 0-absent, 2-present \\
\hline
\end{tabular}

Department of Otolaryngology, 5th Military Hospital, Cracow, Poland Corresponding Author: Anna Salwa, Department of Otolaryngology, 5th Military Hospital, Cracow, Poland, Phone: +48 504818554, e-mail: anna.salwa.05@gmail.com

How to cite this article: Salwa A. Importance of Laryngopharyngeal Reflux in the Incidence of Benign Vocal Folds' Lesions. Int J Phonosurg Laryngol 2019;9(1):6-8.

Source of support: Nil

Conflict of interest: None

\section{Materials and Methods}

The group of respondents consisted of 63 persons with pathologic conditions include Reinke's edema (21 cases), vocal cord polyps (27 cases), and granulomas (15 cases); additionally, the control group has been determined (16 cases). Exclusion criteria from the study group were defined as the following: consumption of proton pump inhibitors (PPI) or $\mathrm{H}_{2}$ blockers and previously diagnosed the GERD or LPR. The control group inclusion criteria were the following: no laryngeal benign or malignant masses in the actual laryngoscopy, no history of previously observed laryngeal tumors, videolaryngoscopy was performed as a routine admission test (mostly patients for the septoplasty procedure). An analysis of videolaryngoscopic findings was performed using the RFS.

\section{Results}

Results confirming the LPR in the RFS scale was observed in $80.95 \%$ of patients with Reinke's edema, $60 \%$ of patients with granuloma of the larynx, and $40.74 \%$ with the vocal cord polyps compared with the control group, where a positive result was achieved in $25 \%$ of patients (Figs 1 and 2).

The absolute value of the correlation coefficient was measured in these groups of patients. The weak positive correlation was

(C) The Author(s). 2019 Open Access This article is distributed under the terms of the Creative Commons Attribution 4.0 International License (https://creativecommons. org/licenses/by-nc/4.0/), which permits unrestricted use, distribution, and non-commercial reproduction in any medium, provided you give appropriate credit to the original author(s) and the source, provide a link to the Creative Commons license, and indicate if changes were made. The Creative Commons Public Domain Dedication waiver (http://creativecommons.org/publicdomain/zero/1.0/) applies to the data made available in this article, unless otherwise stated. 


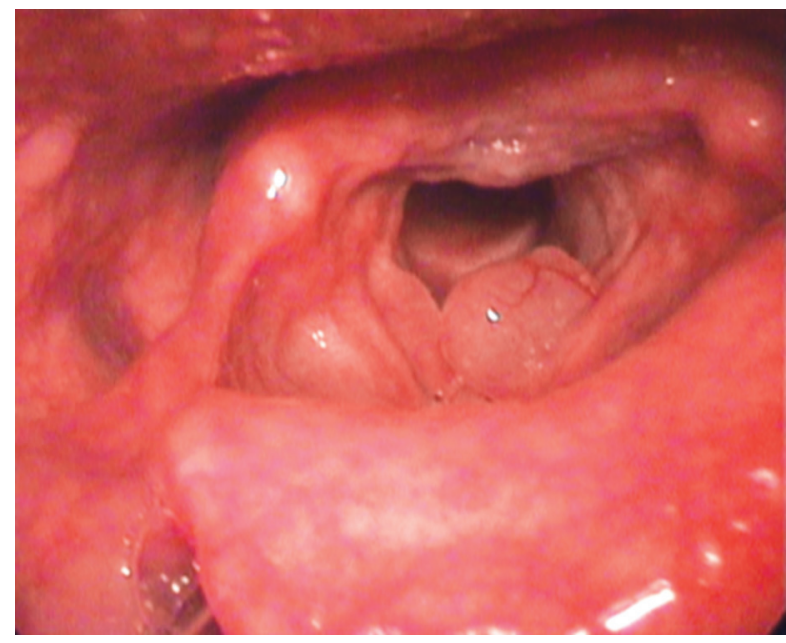

Fig. 1: Reinke's edema (visible posterior commissure hypertrophy and vocal fold edema)

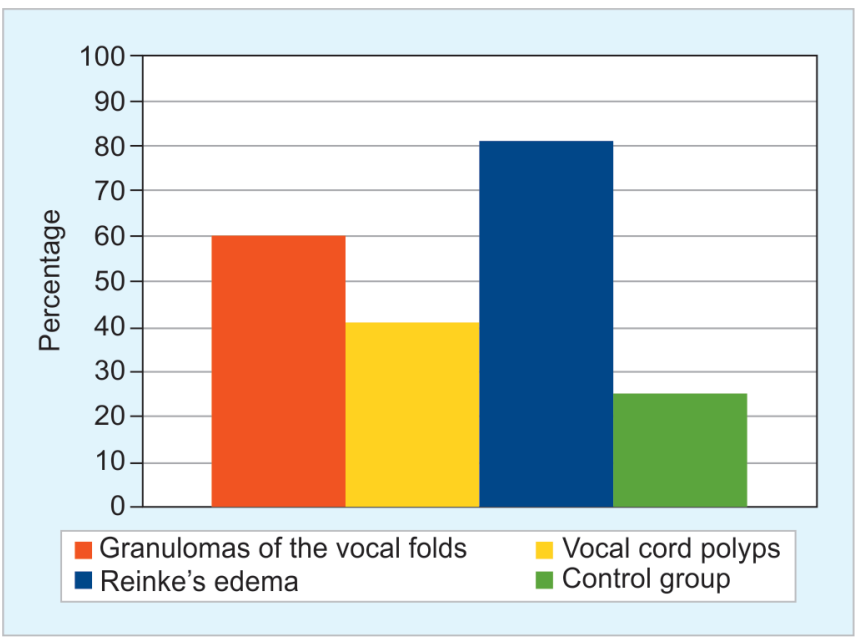

Fig. 3:The presence of laryngopharyngeal reflux in the studied diseases

observed between Reinke's edema and LPR ( $r=0.26, p=0.01)$, and no association was found in the group of granuloma and polyps. The weak negative correlation was observed between the control group and LPR ( $r=-0.24, p=0.02)$, which means that the LPR is less common in the control group than in any other group (Fig. 3).

The most common parameters of the scale RFS noticed in the group of subjects with the positive test result were posterior commissure hypertrophy ( $96.77 \%$ of respondents), vocal fold edema (87.10\%), and subglottic edema (70.97\%).

Further parameters according to the frequency are erythema/ hyperemia of the mucosa (48.39\%), ventricular obliteration (45.16\%), diffuse laryngeal edema (35.48\%), presence of a granuloma/ granulation (25.81\%), and presence of thick endolaryngeal mucus (16.13\%) (Fig. 4).

If we consider the control group subjects who tested positive, also here the most frequently observed changes are changes in hypertrophic posterior commissure, vocal fold edema, and subglottic edema.

\section{Discussion}

In our study, patients suspected of the LPR mainly presented endoscopic signs of inflammation in the posterior section of the larynx,

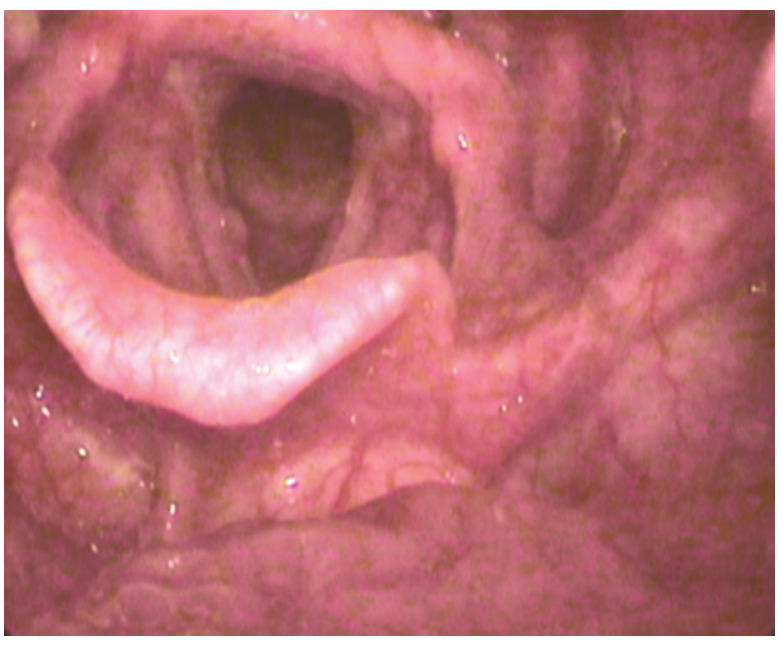

Fig. 2: Vocal cord polyp (visible subglottic edema)

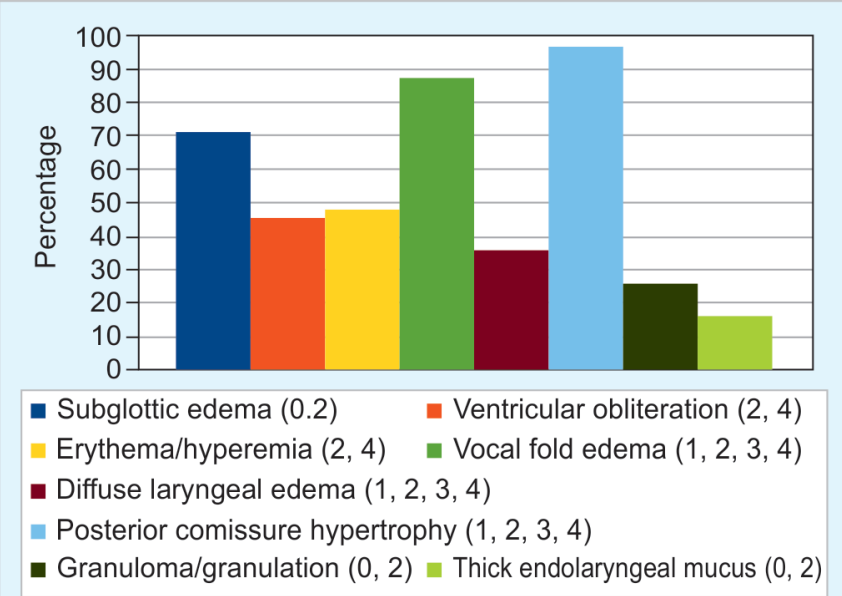

Fig. 4: The incidence of parameters included in the reflux finding score scale

which includes edema, erythema, and hypertrophic changes. ${ }^{1,3,4}$ It is anatomically justified by its proximity to the esophagus.

Acid reflux of gastric contents causes the widening of blood vessels in mucosa and submucosa of the larynx and further edema of the stroma and formation of a transudate. Infiltration by fibroblasts causes fibrosis, further leading to distortion of the structure of the larynx.1,6

As a result of irritation of the mucosa and form an inflammation process, a transudate is collected in the space between the lamina propria and the external elastic lamina of the vocal fold (the Reinke's space), and this all leads to the Reinke's edema. Polyps of the vocal cords are formed by the same reaction in the Reinke's space. It has not been clarified exactly whether reflux is only the cofactor of factors such as overexertion of the voice and tobacco smoking, or is a full etiological factor. ${ }^{1,7}$

Granulomas of the larynx arise as a result of chronic irritation of an edge of the vocal process of the arytenoid cartilage by the acid gastric reflux. The loss of a mucosa of the vocal process creates an inflammatory response and forming ulceration. As a result of repair, processes formed granulation tissue covering an ulcer and then forming a granuloma. The LPR is one of the most important etiological factors for granulomas, which can retreat after treatment with proton pump inhibitors. ${ }^{1,7,8}$ 


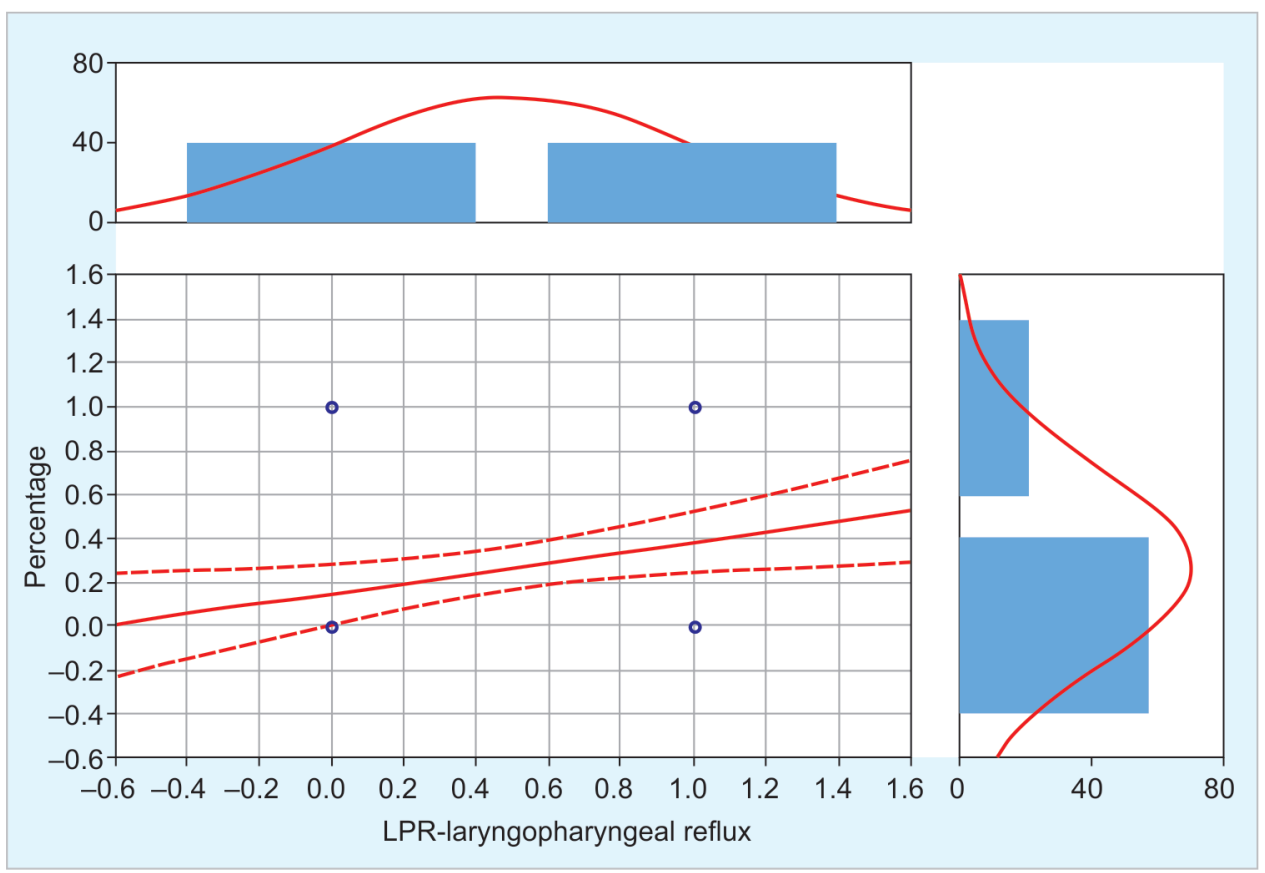

Fig. 5: Correlation values between Reinke's edema and laryngopharyngeal reflux

\section{Conclusion}

The LPR shows a positive coincidence with benign vocal folds lesions. The strongest association is observed in patients with the Reinke's edema (Fig. 5).

\section{References}

1. Skrzypczak W. Inflammatory and neoplastic lesions of the laryngeal mucosa in gastroesophageal reflux disease. Otolaryngol Pol 2011;65(1):66-67. DOI: 10.1016/S0030-6657(11)70631-X.

2. Witt DR, Chen H, Mielens JD, et al. Detection of chronic laryngitis due to laryngopharyngeal reflux using color and texture analysis of laryngoscopic images. J Voice 2014;28(1):98-105. DOI: 10.1016/ j.jvoice.2013.08.015.

3. Ford CN. Evaluation and management of laryngopharyngeal reflux. JAMA 2005;294(12):1534-1540. DOI: 10.1001/jama.294.12.1534.
4. Campagnolo AM, Priston J, Thoen $\mathrm{RH}$, et al. Laryngopharyngeal reflux: diagnosis, treatment, and latest research. Int Arch Otorhinolaryngol 2014;18(2):184-191. DOI: 10.1055/s-0033-1352504.

5. Habermann W, Schmid C, Neumann K, et al. Reflux symptom index and reflux finding score in otolaryngologic practice. J Voice 2012;26(3):e123-e127. DOI: 10.1016/j.jvoice.2011.02.004.

6. Lai Y-C, Wang P-C, Lin J-C. Laryngopharyngeal reflux in patients with reflux esophagitis. World J Gastroenterol 2008;14(28):4523-4528. DOI: 10.3748/wjg.14.4523.

7. Chung JH, Tae K, Lee YS, et al. The significance of laryngopharyngeal reflux in benign vocal mucosal lesions. Otolaryngol Head Neck Surg 2009;141(3):369-373. DOI: 10.1016/j.otohns.2009.05.033.

8. Tsunoda K, Ishimoto S, Suzuki M, et al. An effective management regimen for laryngeal granuloma caused by gastro-esophageal reflux: combination therapy with suggestions for lifestyle modifications. Acta Otolaryngol 2007;127(1):88-92. DOI: 10.1080/00016480600606 665. 